\title{
SOME CHALLENGES IN CROSS-CULTURAL COMPARATIVE RESEARCH
}

\author{
Alex Krouglov \\ Rezekne Academy of Technologies, Latvia \\ University College London, United Kingdom
}

\begin{abstract}
The paper examines the application of qualitative methods in comparative research in a recent international project Industrial Relations in Multilingual Environments at Work (IR MultiLing) which explored how the use of languages other than a dominant language may affect labour relations in various work situations in Europe. The novelty of the current research covered in this paper lies in the fact that it investigates some challenges in developing the best ways of creating an analytical framework when conducting cross-cultural research involving multinational teams of researchers from France, Germany, Hungary, Italy, Spain and the United Kingdom. It also analyses approaches in completing an effective comparison of the contexts and findings across various cultures. Although, there are many examples of qualitative research in literature, this paper aims at exploring some credible approaches in conducting cross-cultural research by international teams of scholars at several key stages: desk research and contextualisation, identifying clear parameters for fieldwork, systematising and comparing findings of the research. The purpose of this research is to provide guidance and some tools to researchers engaged in cross-cultural comparative research using qualitative methods.
\end{abstract}

Keywords: cross-cultural comparative research, labour relations, qualitative methods

\section{Introduction}

Cross-cultural comparative research has always attracted the attention of numerous academics who were interested in researching and comparing various phenomena in several countries or other entities, e.g. different organisations, societies, groups of people, etc. Cultural differences have presented fascinating material for research on one side and numerous challenges on the other. Whatever phenomena researchers decide to study they need to determine whether and how they need to contextualise their research so that researchers in other cultures could fully appreciate the results and value of their findings and analysis. With these points in mind, this paper aims at investigating some challenges in developing the best ways of creating a common analytical framework when conducting cross-cultural research involving multinational teams of researchers from several countries. This research is based on 
qualitative comparisons and relies "on methodological 'procedures' that from the outset make relationalities or relational structures into a subject matter" (Alheit, 2013, 196) which is grounded on Norbert Elias's sociological traditions (1971) further developed by Murphy, Sheard and Waddington (2000) and often described as 'figuration sociology'. It was important for us "that the investigated social reality is understood not as a numerically classifiable collection of characteristics and variables but is instead perceived as web of interdependence of pressures and possibilities; of institutional structures and individual opportunities" (Alheit, 2013, 196).

At the same time, by analysing the work of researchers in a multinational project, the paper indirectly explores whether current globalisation processes have an impact on cross-cultural studies and whether their role and significance may diminish in the current circumstances. There have been numerous studies about the impact of globalisation on various cultural aspects of our everyday life, statements that cultural differences disappear, that the society and the world are becoming more and more homogenous. But is it really happening? Do we really see 'Macdonaldisation' of cultures and the disappearance of cultural differences? If this is true, we can assume that contextualisation is no longer required since there are no culture-specific features, which may be difficult to specify for a representative of another culture in the era of new information technologies (Little, Holmes, Grieco, 2001). Alternatively, perhaps, the situation is becoming even more complex since phenomena or objects spreading across cultures may, for example, preserve their linguistic labels but transform their semantic values. This makes our understanding of different cultures even more complex because the same phenomena or event may have similar linguistic markers in both cultures, but they will contain different semantic meanings or shades of meaning. The current developments and globalisation, which we understand here "as an interconnected whole and the consciousness that a growing number of issues can no longer be addressed solely at the national level" (Jordan, Stråth, Triandafyllidou, 2003, 204), create new challenges for researchers and the need for further or rather deeper contextualisation of their research and findings.

This paper addresses challenges in cross-cultural research in the current circumstances of globalisation and how researchers deal with various issues in comparative research. For this purpose, we will analyse the involvement of an international team of academics and researchers in a recently completed research project Industrial Relations in Multilingual Environments at Work (IR MultiLing, 2015-2016) which will be referred to as project in the current paper. The project considered the implications of the growing presence of 
multilingualism at work in six countries of the European Union: France, Germany, Hungary, Italy, Spain and the United Kingdom. This issue and arising challenges surprisingly remain largely unexplored while the immigration and the movement of people increased significantly across Europe and worldwide. The key question of the current research is to identify the principal challenges for the project team of researchers and explore possible solutions in dealing with some issues of cross-cultural comparative research in the current circumstances.

\section{Methodology}

Our methodology is based on semi-structured online interviews conducted with researchers engaged in the IR MultiLing project after they completed their final report. They were all sociologists, sociolinguists and experts in industrial relations, language policy and planning. For the purpose of this research, eight scholars were selected in six countries with the aim of collecting qualitative data for an in-depth analysis and understanding of the issues researchers face while investigating cross-cultural differences.

An interview guide was developed which incorporated a list of questions and topics to be covered during the interview. For example, on the topic of challenges during desk research and comparative analysis of individual country reports there were the following questions:

- How did you agree the parameters for each country report? Were there any issues?

- How did your national team work on the report?

- What was your part in writing up your country report?

- When all national reports were completed, how did you proceed with comparative report writing? Were there any stages?

- What was the most difficult part in comparative report writing? Why? And how did you overcome those challenges?

These are only some questions included in the guide on the topic mentioned above and provided here as examples. It is important to note that there was some flexibility with regards to phrasing of questions as well as the order in which they were asked, which allowed the participants to lead the interaction in both anticipated and less predictable directions.

All interviews were conducted in English, although interviewees were from five non-English speaking EU countries and many of them were nonEnglish native speakers. Since the author was the only interviewer, there was no 
need in any pre-interview observations or informal and unstructured interviewing because the author participated in the IR MultiLing project as a researcher and knew all academics and the topics and issues the research project investigated. Although, in these circumstances, there were some reservations that an interviewer "can ever be entirely impersonal and neutral data collector", especially in the views of qualitative neo-positivists (King, Harrods, Brooks, 2019, 210). With this point in mind, the author and researcher aimed to minimise personal impact which was achieved by the development of interview guide mentioned above and "strict limits to personal disclosure by the interviewer” (King, Harrods, Brooks, 2019, 211).

All interviews were audio-recorded and later transcribed for analysis as samples and further presented in this paper as S1, S2, S3, etc., while interviewees were coded as R1, R2, R3, etc. and will be mentioned when quotes from their interviews are presented and analysed in this paper.

Semi-structured interviews allowed our informants certain freedom to express their views in their own terms, provide additional details and their personal views on the analysed research and approaches chosen by teams of scholars participating in the project funded by the Directorate General for Employment, Social Affairs and Inclusion of the European Commission.

The collected interviews in the period of 2017-2018 were transcribed and coded. Appropriate themes common for all interviews were identified and selected for further discussion and analysis using the 'constant comparative method' as developed by Glaser and Strauss (1968) which involves two principal stages: "first, multiplication of hypothesis around" our given datum and "second, the consideration as to whether the next datum examined enables any of the previous hypotheses to be eliminated" (Wengraf, 2001, 302).

\section{Data analysis and discussion}

The collected data was analysed in relation to three broad stages of the IR MultiLing project, i.e.:

- desk research/literature review and comparative analysis of industrial relations and the use of languages in six EU countries;

- development of methodology for case studies and data collection;

- comparative analysis at national and international levels and the formulation of recommendations. 
The common theme which transpired through all interviews irrespective of the research stages was contextualisation on one hand and conceptualisation and generalisation on the other. In a way, the first stage of IR MultiLing project research provided specific contexts of each particular country in relation to language attitudes, sociolinguistic parameters as well as historical developments of migration processes and the elaboration of legal norms and legislation related to industrial relations and the use of languages. The research showed striking differences in the interpretation of various phenomena and consequent reflection in the legislation that prompted a number of questions about the comparability of the data. The participants showed their awareness of the issue and reported that they openly discussed it during their project team meetings $(\mathrm{R} 2,4-6)$. It is, therefore, the issue of comparability which was one of the central issues in the collected data of all interviews as well as in the literature on the topic. In relation to this, one of the interviewees (R3) raised the issue of "translatability of terms in industrial relations (IR) since they are all embedded in the culture and history of labour relations development in each country" (S3). R3 was wondering what researchers do if, for example, the terms or phenomena do not exist in other IR systems, that "what may appear to be comparable processes may in fact denote something different or when the concepts under examination do not have a counterpart in the target system, as they are contextbound and culture-bound" (S3).

While other interviewees (R2, R4, R6 and R8) were in principle agreeing that comparability may present certain challenges, especially when comparing specific legislation in the field of industrial relations, however the process of conceptualisation and generalisation may allow researchers to identify either similar patterns or processes which enable them to provide a comparative analysis and present their conclusions. R1 mentioned that "this was one of the most complex projects I had to deal with in view of cultural, linguistic, legal and academic varieties". In fact, comparability was perhaps another issue which overcomplicated the process of research and analysis of the data during the project $(\mathrm{S} 2-4,6,8)$. Each national team of researchers had their own culturespecific approaches and understanding of researched issues (S7). This duality of cultural differences, i.e. cultural differences of researched issues and cultural differences of six research teams, created several new challenges for the entire project team. In a way, comparative research can be presented graphically below (Fig.1), however the project under consideration was even more complex, since it incorporated six countries and six teams of researchers representing those countries, and there were 18 case studies in total or three case studies per each country. It means that the graphical presentation of the research completed 
within the IR MultiLing project will require significant expansion of what was shown in Fig. 1 and must be multiplied by six.

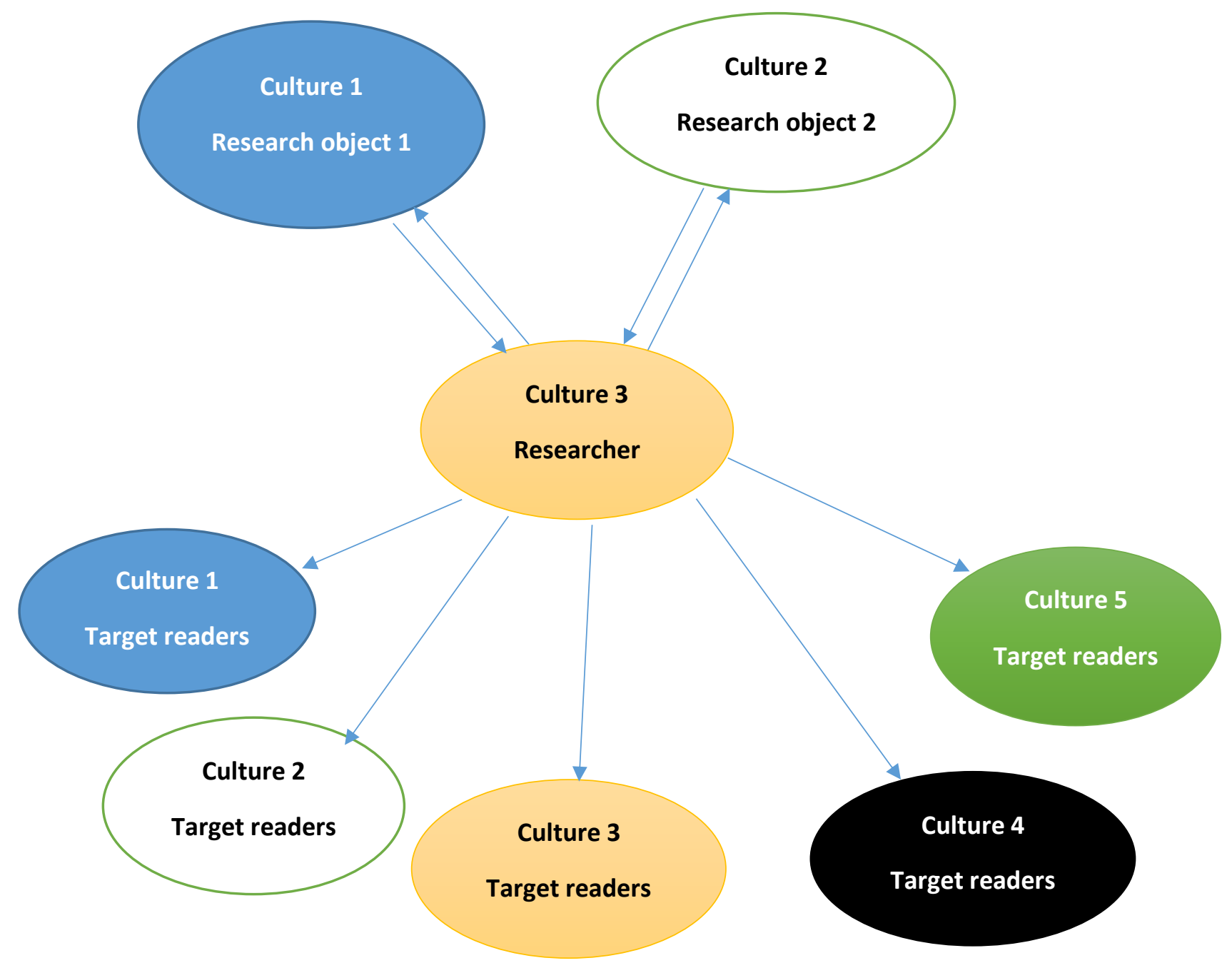

Figure 1 Comparative research model

Participants in our research reported that further challenges in the IR MultiLing comparative study arose from different histories and patterns of immigration as well as 'geography' of languages (vernacular/national vs vehicular/lingua franca languages), differences in language issues due to various dynamics in migration processes and the spread of dialects, regional and minority languages as well as regional cultural differences (S2, 3, 4, 7, 8). For example, several case studies were conducted in Catalonia and one in Northern Ireland. All these factors contributed to challenges in conducting this comparative research and identifying common themes for the purpose of data analysis, drawing conclusions and formulating recommendations. 
However difficult it was for the project teams to draw comprehensive conclusions and provide contextualisation at the national level, it allowed better understanding of relevant migration and language policies, legislation, the role of Trade Unions, language data and trends, national discourse on the topic and approaches in tackling various migration and language issues in industrial relations landscape. "Contextualisation was key for the success of the project, especially during our desk research and sharing the results with each national team of researchers" (R5). Contextualisation also "enabled to have a clearer understanding of commonalities across all six countries involved in the project”(R1). However, some interviewees pointed out certain challenges, e.g. R7 reported "different ways of measuring migration which may affect our perception of migration processes as part of contextualisaion and presenting social context." Many interveiwees (R3-6, 8) also mentioned that although there were numerous international, e.g. UN, and European specific legislative frameworks on languages and their use, the actual application of legal frameworks and their interpretation depended on the country, local cultural traditions and different national regulations. In the case of IR MultiLing project, language specific legislation was taken into consideration and analysed at the initial stages when working on the production of desk reports. In some countries, national constitutions have provisions in terms of language recognition, whereas in others the legal systems are based on Case Law and therefore depend on decisions of adjudicatory tribunals that can be cited as precedent. It is therefore, some desk reports provided examples of court cases.

These differences presented some challenges to the project team and were reflected in national desk reports on the issues of migration and language policy and planning in each country (S2-4, 6, 8) and how they should be reflected in the overal final report $(S 3,6,8)$. The presentation became even more complex when some national reports had to reflect on the point that some countries have provinces or constinuent parts which may have different dynamics $(\mathrm{S} 1,3,7)$. R5 was concerned that not all countries considered in the project are part of the Schengen agreement. The overall report showed differences which reflected either various traditions or current policies or debates on the issue, as well as the push for more convergent immigration policies in the EU. In this respect, many interviewees (R1-5, 7) argued that contextualisation at national levels allowed better understanding of local differences and contributed to the successful conceptualisation and compilation of the overall report covering all six national desk reports and presenting prevailing tendencies in Europe.

All interviewees also reported other challenges they faced at various stages of their project, e.g.: 
- getting access to employers and senior management when exploring opportunities for a case study or when conducting interviews in an organisation (S2, 3, 5, 6, 7);

- explaining the research and its objectives when preparing and conducting case studies (S1, 2, 5, 6, 8);

- ensuring that the discussion is not shifting to cultural issues (S3, 4, 6-8);

- bearing in mind the sensitivity of language issues (S1, 2, 4, 6, 7);

- identifying the language for conducting interviews: whether interviewees would fully understand questions asked in another language and whether there is a need to train interviewers, select interviewers who speak relevant language/s or hire interpreters (mentioned by all interviewees apart from R2 and R4).

All these issues and risks may be pertinent to any cross-cultural research project where representatives of different cultures are involved. There could be another factor which had an impact (usually negative) on research results: when the researcher represented a third culture and failed to establish necessary rapport with the interviewees. This factor was not mentioned in the interviews we had with members of the research teams but was considered when the project team discussed the case studies.

Further challenges arose when the data and 18 samples of case studies were collected from all national teams. How do you analyse those case studies when they represent different organisations and various industries? $(\mathrm{S} 2-4,6,8)$ They also have different proportion of speakers of other non-national languages and different levels of knowledge of national or regional languages $(\mathrm{S} 1,5,6,7)$. In some instances, non-national language speakers spoke the same language, and in some other cases, there were speakers of various languages, as in the case of one multinational company in Hungary where there were speakers of 32 languages representing $38 \%$ of workers, or another example of a call centre in Spain where $75 \%$ working there were migrants. How do you compare the results of case studies when you have so much variation? $(\mathrm{S} 1-4,7)$

The majority of interviewees were concerned about the selection of case studies and how they should be compared (S 1-3, 5, 7, 8): "it was difficult to select case studies and make arrangements for similar organisations to be studied in all countries" (R2); "my main concern was how we compare the results of case studies especially since they represent so many variables" (R8); "each case study seemed to cover the use of languages in completely different industrial relations settings" (R5). It is true that there was a variety of organisations covered in six EU countries which provided a number of ideas 
either in support or against previously conducted desk research by six teams. In fact, case studies of the project confirmed almost all previous conclusions drawn as the result of the desk reports. However, achieving this was not easy as the teams had to combine all case studies, conceptualise their findings and analyse them. When conceptualising and analysing the received data the team came up with the following classification of all case studies across six countries:

1. international hubs: call centres and consultancy companies;

2. a local workforce: production sites and outsourced services;

3. public services: health care;

4. professionals and managers in multinational companies.

Such classification or rather grouping allowed the team to conceptualise their findings and identify main themes in the project (S2-5, 7): "I was surprised to see that the themes became obvious when we completed the classication of case studies" (R7); "when we grouped our case studies we could proceed with identifying major themes - starting with the themes repeated in the majority of case studies and moving to themes characteristic for a particular branch of industry or country" (R4); "I thought that many themes are typical for health care and possibly public services only, however when we analysed other case studies it transpired that many of those themes are relevant for other industries and countries" (R3). It looks that the suggested process of grouping the results according to particular factors or specific features allows for a more productive and efficient way of analysing the collected data and enables better selection of main themes across a variety of case studies or samples.

\section{Conclusions}

The current research showed the importance of awareness of crosscultural issues and identified some challenges researchers may face in this type of research. Current globalisation and Europeanisation processes do not diminish the importance of cross-cultural research. On the contrary, the current research shows that even within the EU where countries are bound by certain common regulations and legal framework, the globalisation of cultures brings new challenges for researchers since various notions, ideas and processes are usually localised or transformed in line with the traditions of a country, society or specific organisation. The issue of comparability has been identified in this research as one of the most challenging one since some compared phenomena may be first presented and analysed in different contexts, e.g. national, regional, local. Particular issues were reported when comparing legal terms. 
Contextualisation remains a critical component at all stages of research in the period of globalisation: from the desk research and identification of specific issues to selection of possible samples, data interpretation, conceptualisation and conclusions. It enables clear specification of common problems and formulation of recommendations as well as identifying key themes or overarching problems for the overal report. Our research confirms that contextualisation is the information about how research access was achieved, why certain research decisions were made, as well as what the benefits were of any intervention. Methodological contextualisation may include both contextualising the current state of the researched situation and its development, the process of conducting fieldwork and contextualising the outcome in terms of generated data (Johns 1991, Johns 2001).

The paper covered only some issues of contextualisation and challenges in cross-cultural research in view of the limitations of the current format. Further analysis of our research findings and other studies in the field will enable us to develop and formulate new approaches and tools for the benefit of researchers engaged in comparative studies.

\section{References}

Alheit, P. (2013). Identifying configurations of higher education: Reflections on concepts that compare complex cultural settings. Studies in the Education of Adults, 45:2, 194-209. DOI: $10.1080 / 02660830.2013 .11661651$

Elias, N. (1971). Sociology of Knowledge: New Perspectives: Part One. Sociology, 5:2, 149168. DOI: $10.1177 / 003803857100500201$

Glaser, B., Strauss, A. (1968). The Discovery of Grounded Theory. London: Weidenfeld and Nicholson.

Johns, G. (1991). Substantive and methodological constraints on behavior and attitudes in organisational research. Organisational Behavior and Human Decision Processes, 49, $80-104$.

Johns, G. (2001). In praise of context: commentary. Journal of Organisational Behavior, 22, $31-42$.

Jordan, B., Stråth, B., Triandafyllidou, A. (2003). Contextualising immigration policy implementation in Europe. Journal of Ethnic and Migration Studies, 29:2, 195-224. DOI: $10.1080 / 1369183032000079594$

King, N., Harrocks, C., Brooks, J. (2019) Interviews in Qualitative Research. London: SAGE Publications Ltd.

Little, S., Holmes, L., Grieco, M. (2001) Calling up culture: Information spaces and information flows as the virtual dynamics of inclusion and exclusion. Information Technology \& People, 14:4, 353-367. DOI:10.1108/09593840110411158

Murphy, P., Sheard, K. and Waddington, I. (2000). Figurational sociology and its application to sport. In J. Coakley \& E. Dunning (Eds.), Handbook of sports studies (92-105). London, SAGE Publications Ltd.

Wengraf, T. (2001). Qualitative Research Interviewing. London: SAGE Publications Ltd. 